\title{
Minimum Steiner Trees in Normed Planes*
}

\author{
Ding-Zhu Du, ${ }^{1,2}$ Biao Gao, ${ }^{2}$ Ronald L. Graham, ${ }^{3}$ \\ Zi-Cheng Liu, ${ }^{4}$ and Peng-Jun Wan ${ }^{2}$ \\ ${ }^{1}$ Computer Science Department, University of Minnesota, \\ Minneapolis, MN 55455, USA \\ ${ }^{2}$ Institute of Applied Mathematics, Chinese Academy of Sciences, \\ Beijing, People's Republic of China
}

\author{
${ }^{3}$ AT\&T Bell Laboratories, Murray Hill, NJ 07974, USA \\ ${ }^{4}$ Computer Science Department, Princeton University, \\ Princeton, NJ 08544, USA
}

\begin{abstract}
A minimum Steiner tree for a given set $X$ of points is a network interconnecting the points of $X$ having minimum possible total length. In this note we investigate various properties of minimum Steiner trees in normed planes, i.e., where the "unit disk" is an arbitrary compact convex centrally symmetric domain $D$ having nonempty interior. We show that if the boundary of $D$ is strictly convex and differentiable, then each edge of a full minimum Steiner tree is in one of three fixed directions. We also investigate the Steiner ratio $\rho(D)$ for $D$, and show that, for any $D, 0.623<\rho(D)<0.8686$.
\end{abstract}

\section{Introduction}

Given a compact, convex, centrally symmetric domain $D$ in the Euclidean plane $E^{2}$, we can define a norm $\|\cdot\|_{D}: E^{2} \rightarrow R$ by setting $\|\bar{x}\|_{D}=\lambda$ where $\bar{x}=\lambda \bar{u}$ and $\bar{u} \in \partial D$, the boundary of $D$. We can then define a metric $d_{D}$ on $E^{2}$ by taking

$$
d_{D}(\bar{x}, \bar{y})=\|\bar{x}-\bar{y}\|_{D}
$$

\footnotetext{
* Part of this work was done while Ding-Zhu Du was at the Computer Science Department, Princeton University and the Center for Discrete Mathematics and Theoretical Computer Science at Rutgers. Supported by NSF under Grant STC88-09648.
} 
Thus, $\partial D=\left\{\bar{x} \mid\|\bar{x}\|_{D}=1\right\}$. The resulting metric space $M=M(D)=\left(E^{2}, d_{D}\right)$ is often called a Minkowski or normed plane with unit disk $D$. We usually suppress the explicit dependence of various quantities on $D$. For a finite subset $X \subset E^{2}$, a minimum spanning tree $S=S(X)$ consists of a collection of segments $A B$ with $A$, $B \in X$ which spans all the points of $X$, and such that the sum of all the lengths $\|A B\|_{D}$ is a minimum. We denote this minimum sum by $L_{\mathrm{M}}(X)$. Further, we define

$$
L_{\mathrm{S}}(X)=\inf _{Y \supseteq X} L_{M}(Y)
$$

where $Y$ ranges over all finite subsets of $E^{2}$ containing $X$. It is not hard to show that there always exists $X^{\prime} \supseteq X$ with $\left|X^{\prime}\right| \leq 2|X|-2$ having $L_{\mathrm{S}}(X)=L_{\mathrm{M}}\left(X^{\prime}\right)$. When equality holds we say that the Steiner tree $T(X)\left(=S\left(X^{\prime}\right)\right)$ is a full Steiner tree for $X$. The minimum spanning tree $S(Y)$ is called a minimum Steiner tree $T(X)$ for $X$. The points of $Y \backslash X$ are usually called Steiner points of $T(X)$; the points of $X$ are known as regular points of $T(X)$.

Minimum Steiner trees have been the subject of extensive investigations during the past 25 years or so (see [3], [8], [12], or [6] for a survey of some of this work). Most of this research has dealt with the Euclidean metric, with much of the remaining work concerned with the $L_{1}$ metric, or, more generally, the usual $L_{p}$ metric. It has been shown, for example, that the determination of $L_{T}(X)$ in general is an NP-complete problem, both for the Euclidean as well as the $L_{1}$ case (see [7] and [6]).

In this note we investigate various properties of minimum Steiner trees for general normed planes $M(D)$. In particular we study the Steiner ratio $\rho(D)$ for $M(D)$, defined by

$$
\rho=\inf _{x} \frac{L_{\mathrm{S}}(X)}{L_{\mathrm{M}}(X)}
$$

Thus, $\rho(D)$ is a measure of how much the total length of a minimum spanning tree can be decreased by allowing additional (Steiner) points. It is known [9] that, for the $L_{1}$ metric (so that $D$ is the square with vertices $( \pm 1,0),(0, \pm 1)$ ), $\rho(D)=\frac{2}{3}$. More recently, it has been shown [4] that, for the Euclidean (or $L_{2}$ ) metric, $\rho(D)=\sqrt{3} / 2$. We show that, for any $D$,

$$
0.623<\rho(D)<0.8686
$$

For prior results on minimum Steiner trees in normed planes, the reader should consult [2], [5], [1], [13], [15] and [16]. This note is organized in the following way. In Section 2 fundamental properties of minimum Steiner trees are presented. In Section 3 some properties of the Steiner ratio are given; in particular, we show that $\rho(D) \geq \inf _{H} \rho(H)$ where $H$ ranges over all unit disks which are hexagons. The main result is proved in Section 4. Finally, we give several conjectures in Section 5. 


\section{Fundamental Properties of Minimum Steiner Trees}

Let $|A B|$ denote the Euclidean length of the segment $A B$, and let $\|A B\|$ denote the length of $A B$ in the norm \|\| determined by $D$, an arbitrary fixed compact, convex, centrally symmetric domain in $E^{2}$.

Theorem 1. Suppose that $\partial D$ is differentiable and strictly convex. Then every full Steiner minimum tree consists of three sets of parallel segments.

The proof of Theorem 1 rests on two lemmas.

Lemma 1. Suppose that $\partial D$ is differentiable. Then every Steiner point in a minimum Steiner tree has degree three. Furthermore, if $A, B$, and $C$ are three distinct points of $\partial D$ and $A^{*}, B^{*}, C^{*}$ is the triangle induced by the three tangent lines to $D$ at $A$, $B$, and $C$, respectively, then $\{O A, O B, O C\}$ forms a minimum Steiner tree for $\{A, B, C\}$ if and only if the origin $O$ is the centroid of $A^{*} B^{*} C^{*}$.

The proof of the first half of Lemma 1 is given in [1] and [10]. (The proof in [1] has the hypothesis that the norm is uniformly convex. This hypothesis is removed in [10].) The proof of the second half of Lemma 1 can be found in [2].

The following lemma is the dual of the fact that one side uniquely determines the other two sides of an equilateral triangle in a "nice" norm (see also [10]).

Lemma 2. Suppose $\partial D$ is differentiable and strictly convex and $A \in \partial D$. Then there exist unique points $B$ and $C$ on $\partial D$ such that $\{O A, O B, O C\}$ forms a minimum Steiner tree for the set $\{A, B, C\}$.

Proof. Let $L$ be the line tangent to $\partial D$ at point $A$. Let $L^{\prime}$ and $L^{\prime \prime}$ be two other lines parallel to $L ; L^{\prime}$ passes through $O$ and $L^{\prime \prime}$ is three times as far from $L$ as $L^{\prime}$ is (see Fig. 1(a)). Suppose that $L^{\prime}$ intersects $\partial D$ at two points $B^{\prime}$ and $C^{\prime}$, and that the two tangent lines of $\partial D$ at $B^{\prime}$ and $C^{\prime}$ intersect $L^{\prime \prime}$ at $B^{\prime \prime}$ and $C^{\prime \prime}$, respectively.

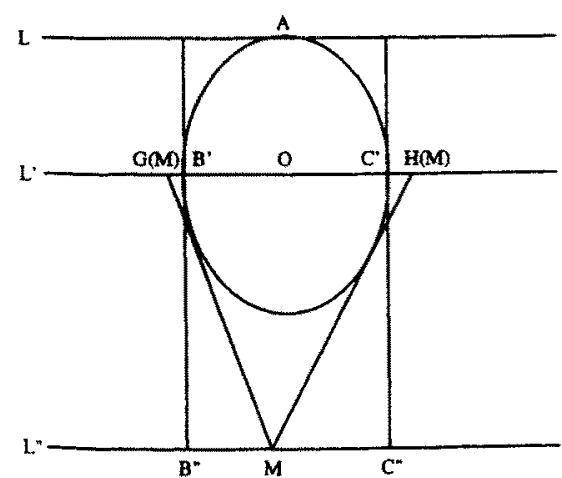

(a)

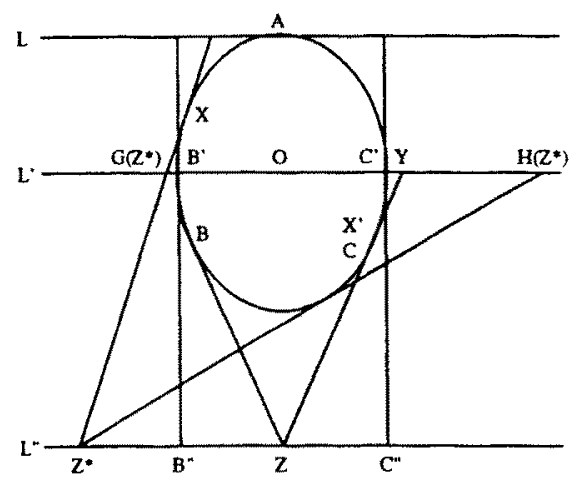

(b)

Fig. 1. Construction of $\{A, B, C\}$. 
Let $M$ be a point on $L^{\prime \prime}$. Through the point $M$ we have two tangent lines to $\partial D$ which intersect $L^{\prime}$ at points $G(M)$ and $H(M)$, respectively. Note that as $M$ goes from $B^{\prime \prime}$ to $C^{\prime \prime}$, the length of the segment $O H(M)$ is decreasing, and the length of $O G(M)$ is increasing. Moreover, $\left|O H\left(B^{\prime \prime}\right)\right|>\left|O G\left(B^{\prime \prime}\right)\right|=\left|O B^{\prime}\right|$ and $\left|O H\left(C^{\prime \prime}\right)\right|=$ $\left|O C^{\prime}\right|<\left|O G\left(C^{\prime \prime}\right)\right|$. Thus, there exists a point $Z$ between $B^{\prime \prime}$ and $C^{\prime \prime}$ such that $|O H(Z)|=|O G(Z)|$. For such a point $Z$, let $B$ and $C$ be the tangent points on lines $Z G(Z)$ and $Z H(Z)$, respectively, and let $L$ intersect $Z G(Z)$ and $Z H(Z)$ at points $E$ and $F$, respectively. It is not hard to see that $O$ is the centroid of triangle $Z E F$. By Lemma $1, O A, O B$, and $O C$ form a minimum Steiner tree for $\{A, B, C\}$. This proves the existence of the required points $B$ and $C$.

To prove uniqueness, suppose $B$ and $C$ satisfy the required hypothesis. Let $Z E F$ be the triangle formed by the three tangent lines to $\partial D$ at $A, B$, and $C$, respectively. By Lemma $1, O$ is the centroid of triangle $Z E F$. Thus, $Z$ must be on line $L^{\prime \prime}$ and $|O G(Z)|$ must equal $|O H(Z)|$. If $Z$ lies between $B^{\prime \prime}$ and $C^{\prime \prime}$, then we have seen that there is just one such $Z$ satisfying $|O G(Z)|=|O H(Z)|$. Next, we show that if $Z=Z^{*}$ is not between $B^{\prime \prime}$ and $C^{\prime \prime}$, then $|O G(Z)| \neq|O H(Z)|$. Without loss of generality, assume that $Z^{*}$ is on the left-hand side of $B^{\prime \prime}$ (see Fig. 1(b)). Then the line $Z^{*} G\left(Z^{*}\right)$ is tangent to $\partial D$ at a point $X$ above the $L^{\prime}$. Let $X^{\prime}$ be the symmetric image of $X$ on $\partial D$, and let the tangent line to $\partial D$ at $X$ intersect $L^{\prime}$ at $Y$. Clearly, $\left|O G\left(Z^{*}\right)\right|=|O Y|<\left|O H\left(Z^{*}\right)\right|$.

Theorem 1 follows at once from Lemmas 1 and 2 .

The dual norm $\|\cdot\|_{D^{*}}$ of a norm $\|\cdot\|_{D}$ is defined by

$$
\|x\|_{D^{*}}=\max _{y} \frac{x^{T} y}{\|y\|_{D}}
$$

It is a well-known fact that $D^{*}$ is the polar dual of $D$, i.e.,

$$
D^{*}=\left\{y: x^{T} y \leq 1 \text { for all } x \in D\right\}
$$

and $D^{* *}=D$. It can be shown (see [2]) that, for triangle $A^{*} B^{*} C^{*}$ in Lemma 1 , $\left\|A^{*} B^{*}\right\|_{D^{*}}=\left\|B^{*} C^{*}\right\|_{D^{*}}=\left\|C^{*} A^{*}\right\|_{D^{*}}$, i.e., $A^{*} B^{*} C^{*}$ is an equilateral triangle in the dual norm. From this observation and Theorem 1, it is easy to show that, for differentiable and strictly convex $D$, any direction determines two unique directions such that three lines respectively in these directions intersect at vertices of an equilateral triangle. We call these directions a consistent triple of directions (with respect to $D$ ).

Suppose that $A, B$, and $C$ are on $\partial D$ such that $O A, O B$, and $O C$ form a consistent triple. Let $A^{\prime}, B^{\prime}$, and $C^{\prime}$ denote the reflections of $A, B$, and $C$ with respect to $O$, respectively. Then $A^{\prime}, B^{\prime}, C^{\prime} \in \partial D$ and the hexagon $A C^{\prime} B A^{\prime} C B^{\prime} A$ is partitioned into six congruent equlateral triangles of side length 1 , by joining each of its six vertices to $O$. The sides of the triangles are in each of the three directions of the consistent triple. 


\section{Relations Between Steiner Ratios}

In this section we investigate relations between Steiner ratios for different norms. The first result is a consequence of Theorem 1.

Lemma 3. Let $\rho_{0}$ be a positive number. Then

(for all norms $\left.\|\cdot\|_{D}, \rho(D) \geq \rho_{0}\right) \Leftrightarrow$ (for all hexagon norms $\left.\|\cdot\|_{H}, \rho(H) \geq \rho_{0}\right)$.

Proof. $\quad \Leftrightarrow$ This direction is clear.

$(\Leftarrow)$ For proving a lower bound, it suffices to restrict our attention to full Steiner trees. First, suppose $\partial D$ (with which determines the norm $\|\cdot\|_{D}$ under consideration) is differentiable and strictly convex. Let $T$ be a full Steiner tree for a point set $P$. By Theorem 1, $T$ consists of three sets of parallel edges (in a consistent triple of directions). Let $\overline{O A}, \overline{O B}$, and $\overline{O C}$ be three vectors of unit length parallel to the edges of $T$. Then $A, B, C \in \partial D$. Let $A^{\prime}, B^{\prime}$, and $C^{\prime}$ be the reflections of $A, B$, and $C$, respectively. Then $A C^{\prime} B A^{\prime} C B^{\prime} A$ is a convex hexagon inscribed in $\partial D$. The length $L_{\mathrm{S}}(P)$ of $T$ in the norm $\left\|^{\prime} \cdot\right\|_{D}$ is equal to the length $L^{\prime}(T)$ of $T$ in the norm $\|\cdot\|_{A C^{\prime} B A^{\prime} C B^{\prime} A}$. By assumption, $L^{\prime}(T) \geq \rho_{0} L_{\mathrm{M}}^{\prime}(P)$, the length of the minimum spanning tree for $P$ under $\|\cdot\|_{A C^{\prime} B A^{\prime} C B^{\prime} A}$. However, since $L_{\mathrm{M}}^{\prime}(P) \geq L_{\mathrm{M}}(P)$, we get $L_{\mathrm{S}}(P) \geq \rho_{0} L_{\mathrm{M}}(P)$.

If $\partial D$ is not differentiable or not strictly convex, we can approximate it by a sequence of differentiable and strictly convex norms approaching $D$. The theorem follows by taking limits.

Lemma 4. Suppose $D$ and $D^{\prime}$ are two unit disks in the plane such that, for some linear transformation $A$ of the plane, we have $A(D)=D^{\prime}$. Then $\rho(D)=\rho\left(D^{\prime}\right)$.

Proof. For any two points $x$ and $y$,

$$
\|x-y\|_{D}=\|A(x-y)\|_{D^{\prime}}=\|A x-A y\|_{D^{\prime}} .
$$

Thus, $A$ is a length-preserving transformation of the $D$-plane to the $D^{\prime}$-plane, and the desired conclusion follows.

Note that every parallelogram $D$ is the image of a square $S$ under a linear transformation. Hwang [9] proved that, for a square $S, \rho(S)=\frac{2}{3}$. Thus, for any parallelogram $D, \sigma(D)=\frac{2}{3}$.

From Lemma 4, we also know that if $D$ and $D^{\prime}$ are similar, then $\rho(D)=\rho\left(D^{\prime}\right)$. Note that $D$ and $D^{\prime}$ are similar if and only if \|\|$_{D}=\lambda\left\|^{\prime} \cdot\right\|_{D^{\prime}}$ for some positive constant $\lambda$. If $\|\cdot\|_{A}=\lambda\left\|_{B}\right\|_{B}$ for a positive constant $\lambda$, then the unit disks $A$ and $B$ are similar and $\rho_{A}=\rho_{B}$.

Lemma 5. Let $\|\cdot\|_{D}=\lambda\|\cdot\|_{D^{\prime}}$ and $D^{\prime} \subseteq D \subseteq D^{\prime \prime}$. Then $\rho(D) \geq \lambda \rho\left(D^{\prime}\right)$.

Proof. Let $\|T\|_{D}$ denote the total length of a tree $T$ under the norm $\|\cdot\|_{D}$. Clearly, $\|T\|_{D^{\prime}} \geq\|T\|_{D} \geq\|T\|_{D^{\prime \prime}}$. A minimum Steiner tree (or a minimum spanning tree) on 
a point set $P$ in the Minkowski plane with norm $\|\cdot\|_{D^{\prime}}$ is denoted by $\operatorname{SMT}_{D^{\prime}}(P)$ (or $\operatorname{MST}_{D}(T)$ ). Then we have

$$
\left\|\operatorname{MST}_{D}(P)\right\|_{D} \leq\left\|\operatorname{MST}_{D^{\prime}}(P)\right\|_{D} \leq\left\|\operatorname{MST}_{D^{\prime}}(P)\right\|_{D^{\prime}}
$$

and

$$
\left\|\operatorname{SMT}_{D}(P)\right\|_{D} \geq\left\|S M T_{D}(P)\right\|_{D^{\prime \prime}} \geq\left\|\operatorname{SMT}_{D^{\prime \prime}}(P)\right\|_{D^{\prime \prime}}=\lambda\left\|S M T_{D^{\prime}}(P)\right\|_{D^{\prime}}
$$

Thus,

$$
\frac{\left\|\mathrm{SNT}_{D}(P)\right\|_{D}}{\left\|\mathrm{MST}_{D}(P)\right\|_{D}} \geq \lambda \frac{\left\|\mathrm{SMT}_{D^{\prime}}(P)\right\|_{D^{\prime}}}{\left\|\mathrm{MST}_{D^{\prime}}(P)\right\|_{D^{\prime}}}
$$

for any point set $P$. It follows that $\rho(D) \geq \lambda \rho\left(D^{\prime}\right)$.

In Lemma 5 choose $D$ to be a regular $2 n$-gon and $D^{\prime}$ and $D^{\prime \prime}$ to be the circumscribed and inscribed circles of $D$, respectively. Then it is known that $\rho(D) \geq \sqrt{3} / 2 \cos (\pi / 2 n)$. (This is a result of Sarrafzadeh and Wong [14].)

\section{Bounds for the Steiner Ratio}

We prove the following theorem in this section.

Theorem 2. For any $D$,

$$
\rho_{0}=0.623 \cdots \leq \rho(D) \leq \frac{\sqrt{13}-1}{3}=0.8685 \cdots
$$

where $\rho_{0}$ is the root of $16 x^{3}-3 x-2=0$ in $(0.5, \infty)$.

Proof. We first prove the lower bound. By Lemma 3, it suffices to consider the case that $D$ is a hexagon. To begin, let us first explain where the equation $16 x^{3}-3 x-2=0$ comes from.

Let $A, B, C, A^{\prime}, B^{\prime}$, and $C^{\prime}$ denote vertices of $D$, and suppose first that they are on a circle with center at the origin $O$. Assume that $2 \alpha=\angle A O B^{\prime}=\angle B^{\prime} O C \geq$ $\angle C O A^{\prime}$. Then the circle with center $O$ and tangent to $A B^{\prime}$ lies inside hexagon $D$ (see Fig. 2). Using Lemma 5 , we choose $D^{\prime}$ and $D^{\prime \prime}$ to be the two circles of radii $\cos \alpha$ and 1 , respectively, with center $O$. Then we obtain

$$
\rho(D) \geq \frac{\sqrt{3}}{2} \cos \alpha
$$

Let $\bar{A} \bar{B} \bar{A}^{\prime} \bar{B}^{\prime}$ be the parallelogram similar to parallelogram $A B A^{\prime} B^{\prime}$ with respect to 


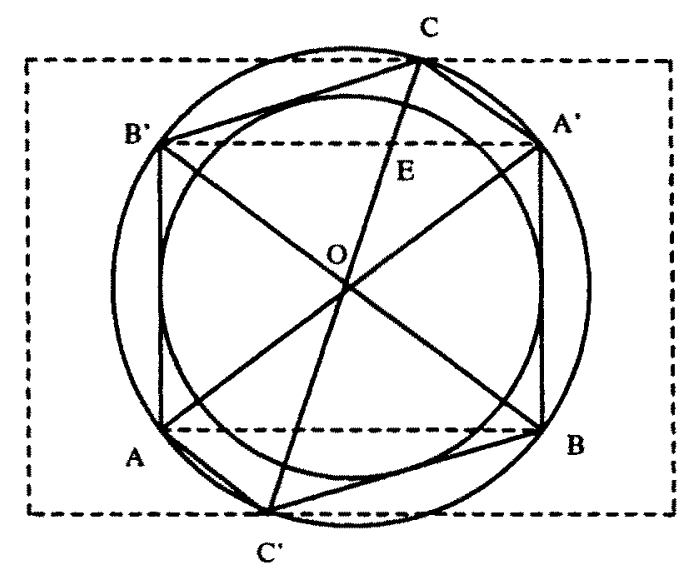

Fig. 2. The hexagon norm $D$.

the origin $O$ and passing through $C$. Let $E$ be the intersection of $O C$ and $B^{\prime} A^{\prime}$. It is easy to see that

$$
\frac{\|\cdot\|_{A B A^{\prime} B^{\prime}}}{\|\cdot\|_{\overline{A B} \bar{A}^{\prime} \bar{B}^{\prime}}}=\frac{|O C|}{|O E|}
$$

Note that $\angle O B^{\prime} A^{\prime}=\angle O A^{\prime} B^{\prime}=\left(180^{\circ}-\angle B^{\prime} O A^{\prime}\right) / 2=\angle A O B^{\prime} / 2=\alpha$. Thus, $\angle O E A^{\prime}=\angle B^{\prime} O C+\angle O B^{\prime} A^{\prime}=3 \alpha$. Therefore, $|O E| /|O C|=\sin \alpha / \sin 3 \alpha$. Again using Lemma 5 , we take $D^{\prime}$ and $D^{\prime \prime}$ to be the parallelograms $A B A^{\prime} B^{\prime}$ and $\bar{A} \bar{B} \bar{A}^{\prime} \bar{B}^{\prime}$. Then we have

$$
\rho(D) \geq \rho\left(A B A^{\prime} B^{\prime}\right) \frac{\sin \alpha}{\sin 3 \alpha}=\frac{2}{3} \frac{\sin \alpha}{\sin 3 \alpha},
$$

since $\rho(P)=\frac{2}{3}$ for a parallelogram $P$. We have now obtained two lower bounds in (1) and (2). Next, we consider what happens if the two lower bounds agree. Set

$$
x=\frac{\sqrt{3}}{2} \cos \alpha=\frac{2}{3} \frac{\sin \alpha}{\sin 3 \alpha} .
$$

Note that $\sin 3 \alpha=\sin \alpha(4 \cos \alpha-1)=\sin \alpha\left(\frac{16}{3} x^{2}-1\right)$. Thus, we have

$$
16 x^{3}-3 x-2=0
$$

Now, let $\alpha_{0}$ denote the angle in $\left(0^{\circ}, 90^{\circ}\right)$ such that

$$
\rho_{0}=\frac{\sqrt{3}}{2} \cos \alpha_{0}=\frac{2}{3} \frac{\sin \alpha_{0}}{\sin 3 \alpha_{0}} .
$$


We consider the general hexagon norm \|\|$_{D}$. Since $D$ is a centrally symmetric hexagon, it is easy to find a centrally symmetric quadratic curve passing through the six vertices of $D$. Since $D$ is convex, this quadratic curve must be either an ellipse or a pair of parallel lines. If it is a pair of parallel lines, then hexagon $D$ degenerates to a parallelogram, which is the image of a square under a linear transformation. In that case, by Lemma 4, we obtain $\rho(D) \geq \frac{2}{3}>\rho_{0}$ (see the remark after Lemma 4). Thus, we can assume that the quadratic curve is an ellipse. Hence, we can linearly transform it into a unit circle. At the same time, the hexagon $D$ is transformed into a centrally symmetric hexagon inscribed in the circle. By Lemma 4, it suffices to study the latter hexagon. Thus, the assumption that $D$ is inscribed in a circle is no loss of generality. Also, we may assume $\angle A O B^{\prime} \geq$ $\angle B^{\prime} O C \geq \angle C O A^{\prime}$. Next, we consider two cases.

Case 1: $\angle A O B^{\prime} \leq 2 \alpha_{0}$. In this case the circle of radius $\cos \alpha_{0}$ with center $O$ lies inside hexagon $D$. So, by Lemma 5 and (3), we have

$$
\rho(D) \geq \frac{\sqrt{3}}{2} \cos \alpha_{0}=\rho_{0} .
$$

Case 2: $\angle A O B^{\prime}>2 \alpha_{0}$. Let $L$ be a linear transformation which transforms $A$ and $B^{\prime}$ to $A$ and $B^{\prime \prime}$, respectively, where $B^{\prime \prime}$ is a point on the unit circle such that $\angle A O B^{\prime \prime}=2 \alpha_{0}$. Suppose that $L$ transforms $C$ to $C^{\prime \prime}$. We claim that $\left|O C^{\prime \prime}\right| \leq 1$ (see Fig. 3). To show this, let us write $O C=\lambda O B^{\prime}+\xi O A^{\prime}$ where $\lambda>0$ and $\xi>0$. Then $O C^{\prime \prime}=\lambda O B^{\prime \prime}+\xi O A^{\prime}$. So,

$$
\begin{aligned}
\left|O C^{\prime \prime}\right|^{2} & =\lambda^{2}\left|O B^{\prime \prime}\right|^{2}+\xi^{2}\left|O A^{\prime}\right|^{2}+2 \lambda \xi\left|O B^{\prime \prime}\right| \cdot\left|O A^{\prime}\right| \cos \angle B^{\prime \prime} O A^{\prime} \\
& =\lambda_{2}+\xi^{2}-2 \lambda \xi \cos 2 \alpha_{0} \\
& \leq \lambda^{2}+\xi^{2}-2 \lambda \xi \cos \angle A O B^{\prime} \\
& =|O C|^{2}=1 .
\end{aligned}
$$

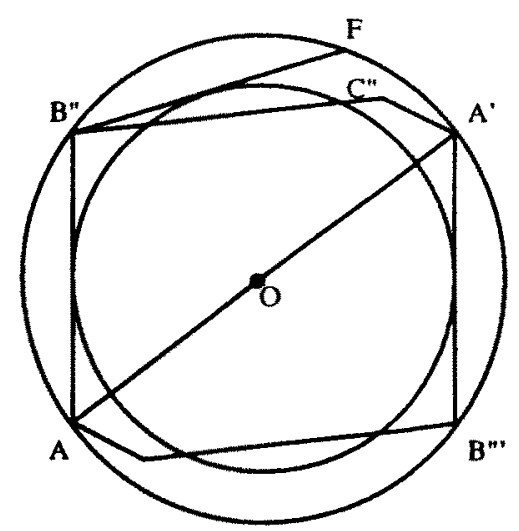

Fig. 3. Proof that $\left|O C^{\prime \prime}\right| \leq 1$. 
Thus, $\left|O C^{\prime \prime}\right| \leq 1$, i.e., $C^{\prime \prime}$ is inside the unit circle. Now, we consider the hexagon $A B^{\prime \prime} C^{\prime \prime} A^{\prime} B^{\prime \prime} C^{\prime \prime \prime}$ where $B^{\prime \prime \prime}$ and $C^{\prime \prime \prime}$ are the images of $B$ and $C^{\prime}$ under the linear transformation $L$. If the circle $C_{2}$ of radius $\cos \alpha_{0}$ and center $O$ lies inside hexagon $A B^{\prime \prime} C^{\prime \prime} A^{\prime} B^{\prime \prime} C^{\prime \prime \prime}$, then, by Lemma 5 , we have

$$
\rho(D)=\rho\left(A B^{\prime \prime} C^{\prime \prime} A^{\prime} B^{\prime \prime \prime} C^{\prime \prime \prime}\right) \geq \frac{\sqrt{3}}{2} \cos \alpha_{0}=\rho_{0} .
$$

Next, we assume that circle $C_{2}$ does not lie inside hexagon $A B^{\prime \prime} C^{\prime \prime} A^{\prime} B^{\prime \prime \prime} C^{\prime \prime \prime}$. Then $C_{2}$ must intersect either $B^{\prime \prime} C^{\prime \prime}$ or $C^{\prime \prime} A^{\prime}$. Choose a point $F$ on the arc between $B^{\prime \prime}$ and $A^{\prime}$ such that $\left|B^{\prime \prime} F\right|=\left|A B^{\prime \prime}\right|$. Let $\bar{A} \bar{B} \bar{A}^{\prime} \bar{B}^{\prime}$ be a parallelogram similar to parallelogram $A B^{\prime \prime} A^{\prime} B^{\prime \prime \prime}$ with respect to the origin $O$ and passing through the point $F$. Then parallelogram $\bar{A} \bar{B} \bar{A}^{\prime} \bar{B}^{\prime}$ contains the hexagon $A B^{\prime \prime} C^{\prime \prime} A^{\prime} B^{\prime \prime \prime} C^{\prime \prime}$. By Lemma 5 , we have

$$
\rho(D)=\rho\left(A B^{\prime \prime} C^{\prime \prime} A^{\prime} B^{\prime \prime \prime} C^{\prime \prime}\right) \geq \frac{2}{3} \frac{\sin \alpha_{0}}{\sin 3 \alpha_{0}}=\rho_{0} .
$$

This completes the proof of the lower bound.

Next, we prove the upper bound. Suppose $D$ is a compact convex centrally symmetric region with center $O$. First, assume that $\partial D$ is strictly convex and differentiable. Let $A, B$, and $C$ be three points on $\partial D$ such that $O A, O B$, and $O C$ form a consistent triple of directions. Let $A^{\prime}, B^{\prime}$, and $C^{\prime}$ denote the reflections of $A, B$, and $C$. Suppose that $E, F$, and $G$ are midpoints of $A B^{\prime}, A C^{\prime}$, and $B^{\prime} C$, respectively. Define $a:=\|O E\|_{D}, b:=\|O F\|_{D}$, and $c:=\|O G\|_{D}$. Note that any point $A$ on $\partial D$ is associated with a unique consistent triple of directions.

Claim 1. There exists a point $A$ on $\partial D$ such that $a \leq b=c$.

Proof. Without loss of generality, assume $a \leq b \leq c$. As $A$ moves to the position of $C^{\prime}$, we have $c \leq a \leq b$. View $(a, b, c)$ as a point in three-dimensional space. The three planes $a=b, b=c$, and $c=a$ partition the first quadrant into six regions. A point going continuously from the region $a \leq b \leq c$ to the region $c \leq a \leq b$ must cross one of boundaries $a=b \geq c, a \leq b=c$, or $b \leq a=c$ (see Fig. 4). Rearranging the labels of points, we obtain $a \leq b=c$ for some point $A$.

Extend $O E, O F$, and $O G$ to intersect $\partial D$ at $E^{\prime}, F^{\prime}$, and $G^{\prime}$, respectively. Let $H$ be the midpoint of $O E^{\prime}$. Draw the line $H^{\prime} H H^{\prime \prime}$ parallel to $C C^{\prime}$ with $H^{\prime}$ and $H^{\prime \prime}$ as intersection points with $\partial D$ (see Fig. 5).

Claim 2. If $a=b \leq c$, then $\left|H^{\prime} H\right| \geq\left|H H^{\prime \prime}\right|$. Moreover, $\left|H^{\prime} H\right|=\left|H H^{\prime \prime}\right|$ if and only if $a=b=c$.

Proof. Connect $F$ and $G$. Then $F G$ is parallel to $C^{\prime} C$ (see Fig. 5). Let $I$ be the intersection of $F G$ and $O E$. Then $I$ is the midpoint of $F G$ and also the midpoint of $O E$. Assume that line $H^{\prime} H^{\prime \prime}$ intersects lines $O F$ and $O G$ at $F^{\prime \prime}$ and $G^{\prime \prime}$, respectively. 


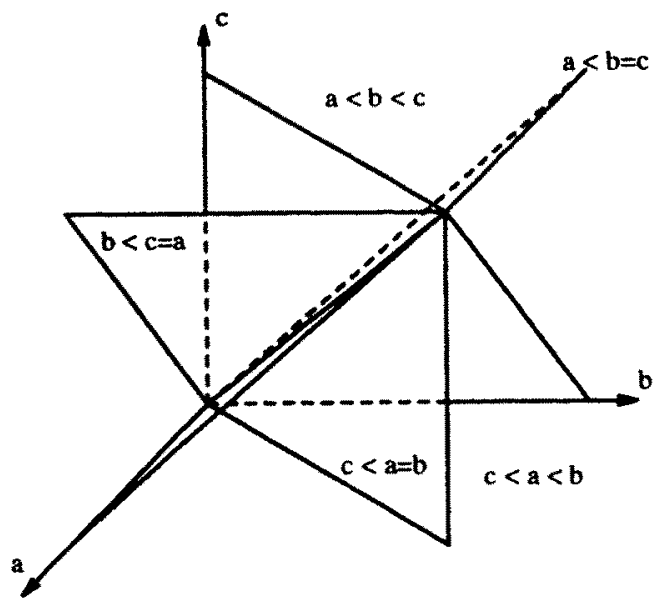

Fig. 4. Cases for Claim 1.

Since $|F I|=|I G|$ and $F G$ is parallel to $H^{\prime} H^{\prime \prime}$, we have $\left|F^{\prime \prime} H\right|=\left|H G^{\prime \prime}\right|$. Moreover,

$$
\frac{|O F|}{\left|O F^{\prime \prime}\right|}=\frac{|O G|}{\left|O G^{\prime \prime}\right|}=\frac{|O I|}{|O H|}=\frac{|O E|}{\left|O E^{\prime}\right|}=\|O E\|_{D}=a
$$

and

$$
\frac{|O F|}{\left|O F^{\prime}\right|}=b, \quad \frac{|O G|}{\left|O G^{\prime}\right|}=c
$$

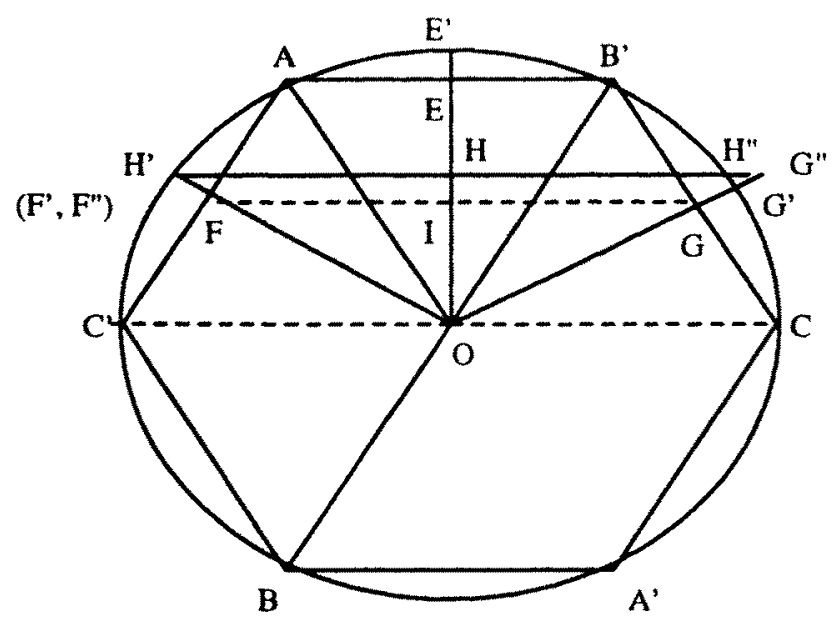

Fig. 5. Construction for Claim 2. 
So, if $a=b \leq c$, then $F^{\prime}, F^{\prime \prime}$, and $H^{\prime}$ are identical and $G^{\prime \prime}$ is on $\partial D$ or on the outside of $D$. Hence $\left|H^{\prime} H\right| \geq\left|H H^{\prime \prime}\right|$. Clearly, $a<c$ if and only if $G^{\prime \prime}$ is on the outside of $D$ if and only if $\left|H^{\prime} H\right|>\left|H H^{\prime \prime}\right|$.

Claim 3. There exists a point $A$ on $\partial D$ such that $a \leq b, a \leq \mathcal{c}$, and $\left|H^{\prime} H\right|=\left|H H^{\prime \prime}\right|$.

Proof. If there exists a point $A$ on $\partial D$ such that $a=b=c$, then, by Claim 2, $\left|H^{\prime} H\right|=\left|H H^{\prime \prime}\right|$. So, we may assume such a point $A$ does not exist. By Claim 1, we can find a point $A$ on $\partial D$ such that $a=b<c$. Now, move $A$ along $\partial D$ to $B$. When $A$ reaches $B$, we have $c=a<b$. We view the points $a, b$, and $c$ in this process as three continuous functions $a(t), b(t)$, and $c(t)$ of a parameter $t$ in an interval $\left[t_{0}, t_{1}\right]$ where $a\left(t_{0}\right)=b\left(t_{0}\right)<c\left(t_{0}\right)$ and $c\left(t_{1}\right)=a\left(t_{1}\right)<b\left(t_{1}\right)$.

First, assume that, for $t \in\left[t_{0}, t_{1}\right], a(t) \leq b(t)$ and $a(t) \leq c(t)$ (see Fig. 6(a)). By Claim 2, $\left|H^{\prime} H\right|>\left|H H^{\prime \prime}\right|$ at $t_{0}$ and $\left|H^{\prime} H\right|<\left|H H^{\prime \prime}\right|$ at $t_{1}$. Thus, there exists $t^{*} \in\left(t_{0}, t_{1}\right)$ such that $\left|H^{\prime} H\right|=\left|H H^{\prime \prime}\right|$ at $t^{*}$. So, the point $A$ corresponding to $t^{*}$ meets our requirements.

Now, we remove the restrictions. Let $t_{2}$ be the largest value $t$ in $\left[t_{0}, t_{1}\right]$ such that $a(t)=b(t)$. Then, for $t \in\left(t_{2}, t_{1}\right], a(t)<b(t)$. Let $t_{3}$ be the smallest value $t$ in $\left(t_{2}, t_{1}\right]$ such that $a(t)=c(t)$. If, for $t \in\left[t_{2}, t_{3}\right), a(t)<c(t)$ (see Fig. 6(b)), then there exists a required $t^{*}$ in $\left[t_{2}, t_{3}\right]$. So, we may assume that, for $t \in\left[t_{2}, t_{3}\right), a(t)>c(t)$. Let $t_{4}$ be the largest $t$ in $\left[t_{0}, t_{3}\right)$ such that $c(t)=\min (a(t), b(t))$. If $b\left(t_{4}\right)=c\left(t_{4}\right)<a\left(t_{4}\right)$ (see Fig. 6(c)), then, by permuting $a, b$, and $c$, we can find a required $t^{*}$ in $\left(t_{4}, t_{3}\right)$. So, we may assume $a\left(t_{4}\right)=c\left(t_{4}\right)<b\left(t_{4}\right)$. Now, the interval $\left[t_{0}, t_{4}\right]$ has the same

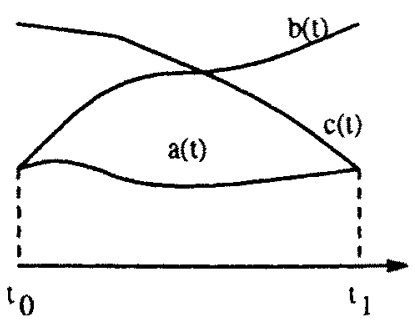

(a)

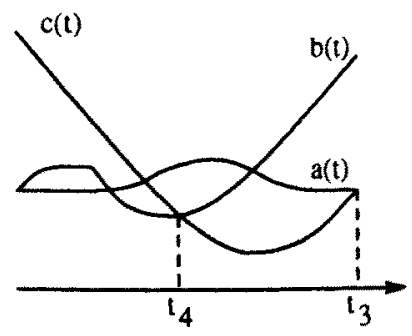

(c)

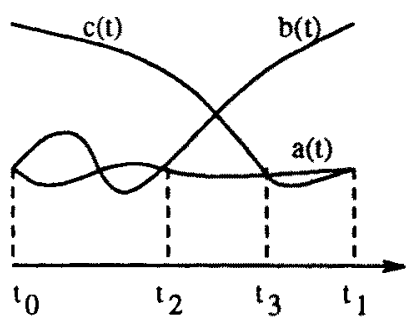

(b)

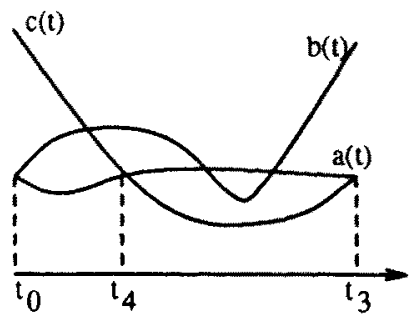

(d)

Fig. 6. Various cases in Claim 3. 
property that the interval $\left[t_{0}, t_{1}\right]$ has. This means that we can either find a required $t^{*}$ or find a smaller interval having the same property as $\left[t_{0}, t_{1}\right]$. Note that

$$
\begin{aligned}
& \left\{t \in\left[t_{0}, t_{1}\right] \mid a(t)=b(t)<c(t)\right\}=\left\{t \in\left[t_{0}, t_{1}\right] \mid a(t)=b(t) \leq c(t)\right\}, \\
& \left\{t \in\left[t_{0}, t_{1}\right] \mid a(t)=c(t)<b(t)\right\}=\left\{t \in\left[t_{0}, t_{1}\right] \mid a(t)=c(t) \leq b(t)\right\} .
\end{aligned}
$$

Since these are closed sets, there exists a minimum distance between them. Let $s_{0}$ and $s_{1}$ be the two values respectively in the two sets such that $\left|s_{0}-s_{1}\right|$ achieves its minimum value. Then, applying the above argument to the interval with endpoints $s_{0}$ and $s_{1}$, we can find a required $t^{*}$ in the interval.

Assume $A$ satisfies Claim 3. Next, we consider three cases.

Case 1: $a \geq \sqrt{3} / 2$. Consider $P_{1}=\{A, B, C\}$. Then

$$
\left\|\operatorname{SMT}\left(P_{1}\right)\right\|_{D} \leq\|A O\|_{D}+\|B O\|_{D}+\|C O\|_{D}=3
$$

and $\left\|\operatorname{MST}\left(P_{1}\right)\right\|_{D} \geq 4 a$. Thus,

$$
\rho(D) \leq \frac{\left\|\operatorname{SMT}\left(P_{1}\right)\right\|_{D}}{\left\|\operatorname{MST}\left(P_{1}\right)\right\|_{D}} \leq \frac{3}{4 a} \leq \frac{\sqrt{3}}{2}<\rho_{1} .
$$

Case 2: $a \leq(\sqrt{13}-2) / 2$. Consider $P_{2}=\left\{O, A, C^{\prime}, B\right\}$. Then

$$
\left\|\operatorname{SMT}\left(P_{2}\right)\right\|_{D} \leq 1+2 a
$$

and $\left\|\operatorname{MST}\left(P_{2}\right)\right\|_{D}=3$. Thus,

$$
\rho(D) \leq \frac{\left\|\operatorname{SMT}\left(P_{2}\right)\right\|_{D}}{\left\|\operatorname{MST}\left(P_{2}\right)\right\|_{D}} \leq \frac{1+2 a}{3} \leq \rho_{1} .
$$

Case 3: $(\sqrt{13}-2) / 2 \leq a \leq \sqrt{3} / 2$. Let $f(a)=a+3 / 4 a$. Then, for

$$
a \leq \sqrt{3} / 2, f^{\prime}(a)=1-3 / 4 a^{2} \leq 0 .
$$

Hence, $f(a) \leq f((\sqrt{13}-2) / 2)=2 \rho_{1}$ for $(\sqrt{13}-2) / 2 \leq a \leq \sqrt{3} / 2$. Define $z:=$ $\left\|H^{\prime} H\right\|_{D}=\left\|H H^{\prime \prime}\right\|_{D}$.

If $a \leq z$, then consider $P_{3}=\left\{O, A, C^{\prime}\right\}$. Let $J$ be the intersection point of $O H^{\prime}$ and $B A$. It is easy to show that $J C^{\prime}$ is parallel to $O H^{\prime \prime}$ and

$$
\left\|J C^{\prime}\right\|_{D}=\|J O\|_{D}=\frac{|J O|}{\left|O H^{\prime}\right|}=\frac{\left|O C^{\prime}\right|}{\left|H^{\prime} H^{\prime \prime}\right|}=\frac{1}{2 z} .
$$




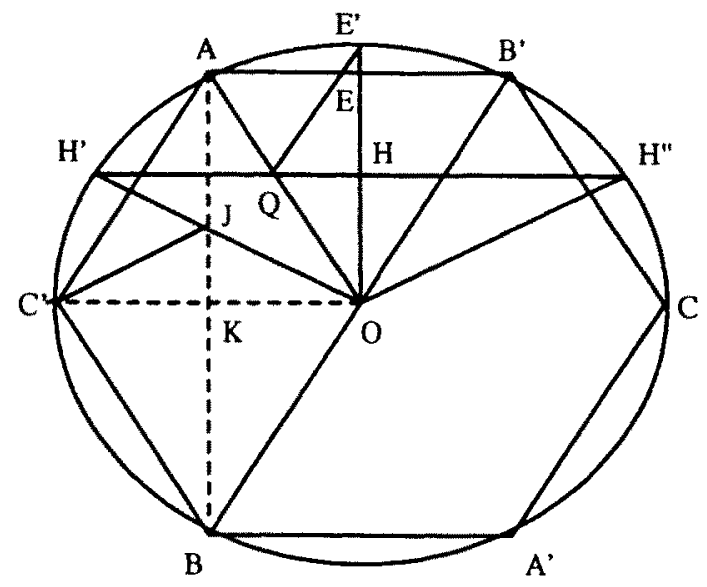

Fig. 7. Locating the point $K$ in proof of upper bound.

Let $K$ be intersection of $O C^{\prime}$ and $A B$ (see Fig. 7). Then

$$
\|J A\|_{D}=a-\frac{|J K|}{\left|O E^{\prime}\right|}=a-\frac{|J K|}{2|O H|}=a-\frac{|J O|}{2\left|O H^{\prime}\right|}=a-\frac{1}{4 z} .
$$

So,

$$
\left\|\operatorname{SMT}\left(P_{3}\right)\right\|_{D} \leq\|J A\|_{D}+\left\|J C^{\prime}\right\|_{D}+\|J O\|_{D} \leq a+\frac{3}{4 z} \leq a+\frac{3}{4 a} \leq 2 \rho_{1}
$$

and $\left\|\operatorname{MST}\left(P_{3}\right)\right\|_{D}=2$. Thus, $\rho(D) \leq \rho_{1}$.

If $a>z$, then consider $P_{4}=\left\{O, E^{\prime}, H^{\prime}\right\}$. Since $\left|H^{\prime} H\right|=\left|H H^{\prime \prime}\right|$ and $\left|H E^{\prime}\right|=$ $|H O|, H^{\prime} O H^{\prime \prime} E^{\prime}$ is a parallelogram. It follows that $\left\|H^{\prime} E^{\prime}\right\|_{D}=1$ and

$$
\left\|\operatorname{MST}\left(P_{4}\right)\right\|_{D}=2
$$

Let $Q$ be the intersection of $O A$ and $H^{\prime} H$. Then triangles $O B A$ and $Q E^{\prime} O$ are similar. So, $Q E^{\prime}$ is parallel to $O B$ and

$$
\left\|Q E^{\prime}\right\|_{D}=\|Q O\|_{D}=\frac{\left|O E^{\prime}\right|}{|A B|}=\frac{1}{2 a}
$$

Moreover,

$$
\left\|Q H^{\prime}\right\|_{D}=z-\frac{|Q H|}{\left|O C^{\prime}\right|}=z-\frac{|Q O|}{2|O A|}=z-\frac{1}{4 a}
$$


So,

$$
\left\|\operatorname{SMT}\left(P_{4}\right)\right\|_{D} \leq z+\frac{3}{4 a} \leq a+\frac{3}{4 a} \leq 2 \rho_{1} .
$$

Thus, $\rho(D) \leq \rho_{1}$.

So far, we have proved the upper bound for $\partial D$ being strictly convex and differentiable. Now, we consider the general case. When $\partial D$ is not strictly convex or not differentiable, we can use a sequence of strictly convex and differentiable norms to approach it from its interior. For each norm in the sequence, we can find $A, B, C$ such that $O A, O B, O C$ form a consistent triple of directions and Claim 3 holds. Since sequences $\{A\},\{B\}$, and $\{C\}$ all lie in the compact set $D$, we can find a subsequence of the norms such that the corresponding subsequences of $\{A\}$, $\{B\}$, and $\{C\}$ all converge. Assume that they converge to $A^{*}, B^{*}$, and $C^{*}$, respectively. Then $O A^{*}, O B^{*}$, and $O C^{*}$ form a consistent triple of directions for $D$ and Claim 3 also holds for them. So, the upper bound $\rho_{1}$ also holds for general norms.

\section{Discussion}

Motivated by the above work, we make the following conjectures.

Conjecture 1. For any norm $\|\cdot\|_{D}, \frac{2}{3} \leq \rho(D) \leq \sqrt{3} / 2$. Moreover, $\rho(D)=\frac{2}{3}$ if and only if $D$ is a parallelogram and $\rho(D)=\sqrt{3} / 2$ if and only if $D$ is an ellipse.

Conjecture 2. Let $D^{\prime}$ be a regular $2 n$-gon. Then, for any centrally symmetric $2 n$-gon $D, \rho(D) \leq \rho\left(D^{\prime}\right)$.

Conjecture 3. For any norm $\|\cdot\|_{D}, \rho(D)=\rho\left(D^{*}\right)$, where $\|\cdot\|_{D^{*}}$ is the dual norm to $\|\cdot\|_{D}$

Conjecture 4. In any normed plane, the Steiner ratio can be achieved by a set of at most four points.

There is a computational method for verifying the lower bound in Conjecture 1. With this method, we have been able to prove that if $|P| \leq 6$, then, in any normed plane, $L_{S}(P) \geq\left(\frac{2}{3}\right) L_{\mathrm{M}}(P)$ (see the Appendix).

For the upper bound in Conjecture 1, Liu and Du [11] proved that it holds for $L_{p}$ norms.

It is easy to show that Conjecture 2 holds for $n \leq 3$. In fact, for $n=2, \rho(D)=\frac{2}{3}$. For $n=3$, it is not hard to show that $\rho(D) \leq \frac{3}{4}$ (see the argument after Lemma 6 in the Appendix). Moreover, from the result at the end of Section 3, $\rho\left(D^{\prime}\right) \geq \frac{3}{4}$ for a regular hexagon $D^{\prime}$. Thus, for $n=3, \rho(D) \leq \frac{3}{4}=\rho\left(D^{\prime}\right)$.

Conjecture 2 implies the upper bound in Conjecture 1 . In fact, any centrally symmetric convex domain can be approximated by a sequence of centrally 
symmetric polygons. Applying Conjecture 2 to these polygons and taking limits, we obtain the upper bound in Conjecture 1.

In fact, we believe Conjecture 3 holds in any normed space.

\section{Acknowledgment}

The authors wish to express their appreciation to Professor Frank Morgan for his insightful comments on an earlier draft of this paper.

\section{Appendix}

Let $\overline{O A}, \overline{O B}$, and $\overline{O C}$ be three vectors satisfying

$$
a \overline{O A}+b \overline{O B}+\overline{C C}=0
$$

for three positive numbers $a, b$, and $c$, where we assume that no two of $\overline{O A}, \overline{O B}$, and $\overline{O C}$ are linearly dependent. In general, let $\overline{O X^{\prime}}$ denote $-\overline{O X}$. Form the centrally symmetric hexagon $H$ by joining (in order) $A C^{\prime} B A^{\prime} C B^{\prime} A$.

Lemma 6. $H$ is convex iff $a, b$, and $c$ satisfy the triangle inequality, i.e.,

$$
a+b \geq c, \quad b+c \geq a, \quad c+a \geq b .
$$

Proof. Note that

$$
\begin{aligned}
& \overline{O A^{\prime}}=\left(\frac{b}{a}\right) \overline{O B}+\left(\frac{c}{a}\right) \overline{O C}, \\
& \overline{O B^{\prime}}=\left(\frac{c}{b}\right) \overline{O C}+\left(\frac{a}{b}\right) \overline{O A}, \\
& \overline{O C^{\prime}}=\left(\frac{a}{c}\right) \overline{O A}+\left(\frac{b}{c}\right) \overline{O B} .
\end{aligned}
$$

Thus, $H$ is convex iff

$$
\frac{b}{a}+\frac{c}{a} \geq 1, \quad \frac{c}{b}+\frac{a}{b} \geq 1, \quad \frac{a}{c}+\frac{b}{c} \geq 1
$$

The norm with $H$ as its unit disk (generated by $\overline{O A}, \overline{O B}$, and $\overline{O C}$ ) is denoted by $\|\cdot\|_{a b c}$ since, by Lemma 4 , the Steiner ratio for it only depends on $a, b$, and $c$. Denote $\rho(H)$ by $\rho(a, b, c)$. Note that $\|O A\|_{a b c}=\|O B\|_{a b c}=\|O C\|_{a b c}=1$ and $\|A B\|_{a b c}=\|B C\|_{a b c}=\|C A\|_{a b c}=2$. Hence, a minimum Steiner tree on $\{A, B, C\}$ 


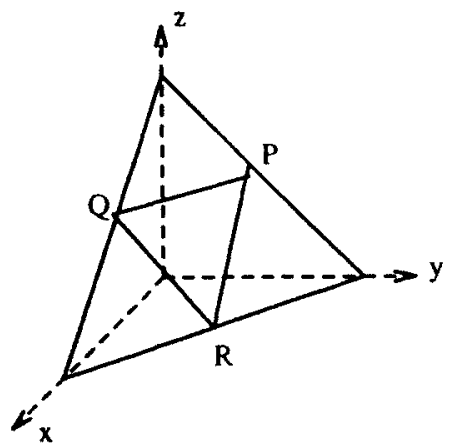

Fig. 8. Set of $(x, y, z)$ satisfying triangle inequality and $x+y+z=1$.

has length at most 3 and a minimum spanning tree on $\{A, B, C\}$ has length 4 . This implies $\rho(a, b, c) \leq \frac{3}{4}$.

Since $\rho(a, b, c)=\rho(k a, k b, k c)$ for $k>0$, we can assume without loss of generality that $a+b+c=1$. The set of all $(a, b, c)$ satisfying the triangle inequality and $a+b+c=1$ forms a triangle with vertices $P=\left(\frac{1}{2}, \frac{1}{2}, 0\right), Q=\left(\frac{1}{2}, 0, \frac{1}{2}\right)$, and $R=\left(0, \frac{1}{2}, \frac{1}{2}\right)$ (see Fig. 8). On the boundary, one of the inequalities of (6) holds with equality; in this case $H$ degenerates to a parallelogram, and so $\rho(H)=\frac{2}{3}$. Thus, $\rho(a, b, c)=\frac{2}{3}$ on the boundary of $P Q R$. Furthermore, $\rho\left(\frac{1}{3}, \frac{1}{3}, \frac{1}{3}\right)=\frac{3}{4}$, which is a maximum value $\rho$ can have over the triangle $P Q R$.

It is easy to verify the truth of Conjecture 1 for sets of at most four points. In fact, let $P$ be a set of $n$ points and let $L_{\mathrm{S}}(P)$ and $L_{\mathrm{M}}(P)$ be lengths of its Steiner minimum tree and minimum spanning tree, respectively. We can prove

$$
L_{\mathrm{S}}(P) \geq(n / 2(n-1)) L_{\mathrm{M}}(P)
$$

in the following way. We first "inflate" the edges of a Steiner minimum tree $T$ on $P$ to have width $\varepsilon$. Thus, $T$ becomes a polygonal region with a boundary. Suppose that $A_{1}, \ldots, A_{n}$ are regular points labeled in counterclockwise order on the boundary. Consider $n$ spanning trees each of which is obtained by deleting an edge from the cycle $A_{1} A_{2} \cdots A_{n} A_{1}$. The total length of these $n$ spanning trees is $n-1$ times the length of the cycle. Moreover, the length of the cycle is less than $2 L_{\mathrm{S}}(P)$. Therefore, $2(n-1) L_{\mathrm{S}}(P) \geq n L_{\mathrm{M}}(P)$. For $n \leq 4$, it follows that $L_{\mathrm{S}}(P) \geq \frac{2}{3} L_{\mathrm{M}}(P)$.

We next introduce a general algebraic method for verifying the conjecture for small $n$. Our method is motivated by recent work of $\mathrm{Du}$ and Hwang [4]. While the main result in [4] is the proof that $\rho(D)=\sqrt{3} / 2$ for the Eucidean plane, much of the proof techniques carry over with no change to general normed planes. In particular, the following result holds. For a general unit disk $D$, let us call a tree a full Steiner tree if, by varying its edge lengths, it can occur as a full minimum Steiner tree for the resulting set of endpoints. For a full Steiner tree $T$, let $P(T)$ denote its endpoints (i.e., vertices of degree one) and let $L(T)$ denote its length (under $\|\cdot\|_{D}$ ). 

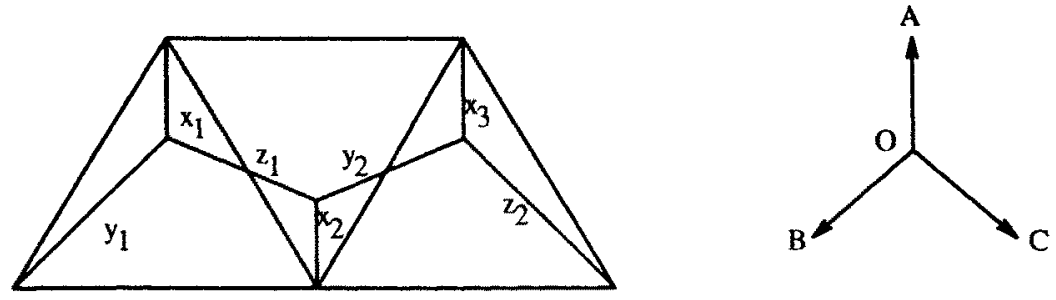

Fig. 9. An example.

Lemma 7 [4]. Suppose $\partial D$ is differentiable and strictly convex, and $C$ is a consistent triple of directions for $D$. Let $\mathscr{T}$ denote the set of full Steiner trees $T$ with edges in the directions of $C$ and with $|P(T)| \leq n$. Then the minimum value of $L_{\mathrm{S}}(T) / L_{\mathrm{M}}(T)$ is achieved by trees $T$ with $P(T)$ being the vertex set of a union $U$ of at most $n-2$ congruent equilateral triangles (with edges in the directions of $C$ ) so that all vertices of $T$ are on the boundary of $U$ (see Fig. 9).

From Lemma 7 and the proof of Lemma 3, we can obtain the following.

Theorem 3. The inequality

$$
L_{\mathrm{S}}(P) \geq \rho_{0} L_{\mathrm{M}}(P)
$$

holds for every norm and every set $P$ of at most $n$ points if and only if it holds for every hexagon norm $H$ and every vertex set $V$ of a union $U$ of at most $n-2$ congruent equilateral triangles such that all the vertices of $V$ lie on the boundary of $U$, and $a$ full Steiner tree for $V$ exists with all edges in the diagonal directions of $H$.

Theorem 3 suggests the following way for verifying this bound for small sets $P$.

Consider a norm $\|\cdot\|_{a b c}$. Given any point set satisfying the hypotheses of Theorem 3, and a full Steiner topology, we compute the length $L_{S}^{\prime}(P)$ of the Steiner tree for $P$ under $\|\cdot\|_{a b c}$ as a function of $a, b$, and $c$ as follows:

1. Choose diagonal directions $\overline{O A}, \overline{O B}, \overline{O C}$ all meeting at $120^{\circ}$ angles so that $\overline{O A}, \overline{O B}, \overline{O C}$ satisfy (5). The corresponding (congruent) equilateral triangles $A C^{\prime} O, C^{\prime} B O, B A^{\prime} O$, etc., have side length 1 (where $X^{\prime}$ denotes $-X$ ).

2. Take all the Euclidean lengths of the edges of $T$ to be variables. For each of the sides of the associated equilateral triangles, we write down a linear equation involving these Euclidean edge lengths (since, under $\|\cdot\|_{a b c}$, all these sides have length 1). If $P$ has $n$ points, then we have $2 n-3$ variables and also $2 n-3$ equations.

3. Represent the length $L=L_{S}^{\prime}(P)$ as a sum of the $\|\cdot\|_{a b c}$-lengths of all the edges. This gives one more linear equation in the Euclidean edge lengths. Since this system of linear equations has a nonzero solution, the determinant of the coefficient matrix must vanish. This allows us to express $L$ as a function $L(a, b, c)$ of $a, b$, and $c$. The conjecture then follows for $\|\cdot\|_{a b c}$ for sets of size $n$ if it is true that $L(a, b, c) \geq \frac{2}{3}(n-1)$. 
An example may clarify the process. Let $n=5$ and consider the Steiner tree topology illustrated in Fig. 9. Thus, assuming $x_{2} \leq y_{1}, x_{2} \leq z_{2}$, we obtain the following linear equations:

$$
\begin{aligned}
& L=a\left(x_{1}+x_{2}+x_{3}\right)+b\left(y_{1}+y_{2}\right)+c\left(z_{1}+z_{2}\right), \\
& 1=a x_{1}+b y_{1}, \\
& 1=a\left(x_{1}+x_{2}\right)+c z_{1}, \\
& 1=b\left(y_{1}-x_{2}\right)+c\left(z_{1}+x_{2}\right), \\
& 1=c\left(z_{1}+x_{1}-x_{3}\right)+b\left(y_{2}-x_{1}+x_{3}\right), \\
& 1=a x_{3}+c z_{2}, \\
& 1=a\left(x_{2}+x_{3}\right)+b y_{2}, \\
& 1=b\left(y_{2}+x_{2}\right)+c\left(z_{2}-x_{2}\right) .
\end{aligned}
$$

If we order the variables as $\left(x_{1}, y_{1}, z_{1}, x_{2}, y_{2}, z_{2}, x_{3}\right)$, we obtain the equation

$$
\operatorname{det}\left(\begin{array}{cccccccc}
L & a & b & c & a & b & c & a \\
1 & a & b & 0 & 0 & 0 & 0 & 0 \\
1 & a & 0 & c & a & 0 & 0 & 0 \\
1 & 0 & b & c & c-b & 0 & 0 & 0 \\
1 & c-b & 0 & c & 0 & b & 0 & b-c \\
1 & 0 & 0 & 0 & 0 & 0 & c & a \\
1 & 0 & 0 & 0 & a & b & 0 & a \\
1 & 0 & 0 & 0 & b-c & b & c & 0
\end{array}\right)=0
$$

A straightforward computation shows that $L=3$ is the only solution to the above equation.

For $n=5$, the only such functions $L=L(a, b, c)$ which can arise from various topologies for $T$ (besides the constant function 3 ) are

$$
3+\frac{(a-c+b)(b-a+c)}{4 a b-b c+b^{2}-a^{2}+a c}
$$

and the expressions which can be obtained from this by permuting $a, b$, and $c$ among themselves. 
For $n=6$, all such possible functions are given by

$$
\begin{aligned}
& 4+\frac{1-2 u}{7-2 u}, \\
& 4+\frac{2(b+c-a)}{2(b+c)+a} \\
& 4+\frac{(1-u)(7-2 w)}{u-2 w+3} \\
& 3.5+\frac{3(1-u)}{2(1+u)} \\
& 5+\frac{u w+w v+u v+2+3 b / a}{5+u+v+w}
\end{aligned}
$$

(and the functions obtained by permuting $a, b$, and $c$ ), where $u=(c-b) / a, v=$ $(a-c) / b$, and $w=(b-a) / c$.

It is interesting to note that all of these functions are "pseudoconcave." (We say the function $f: \mathbb{R} \rightarrow \mathbb{R}$ is pseudoconcave if, for any $x$ and $y$ and $0<\lambda<1$, $f(\lambda x+(1-\lambda) y) \geq \min (f(x), f(y))$. This pseudoconcavity implies that each of the functions assumes its minimum value on the boundary of the triangle $P Q R$ in Fig. 8 . However, we have already observed that, on the boundary, each such function has a value at least $\frac{2}{3}(n-1)$. Hence, we have proved

Corollary 1. If $|P| \leq 6$, then, for any norm,

$$
L_{S}(P) \geq \frac{2}{3} L_{M}(P) .
$$

The above proof suggests that we may be able to establish the pseudoconcavity of these functions $L(a, b, c)$ without having to compute them explicitly. We hope to return to this approach in the near future.

\section{References}

1. M. Alfaro, M. Conger, K. Hodges, A. Levy, R. Kochar, L. Kuklinski, Z. Mahmood, and K. von Haam, The structure of singularities of $\Phi$-minimizing networks in $R^{2}$, Pacific $J$. Math. 149 (1991), 201-210.

2. G. D. Chakerian and M. A. Ghadehari, The Fermat problem in Minkowski space, Geom. Dedicata 17 (1985), 227-238.

3. E. J, Cockayne, On the Steiner problem, Canad. Math. Bull. $10(1967), 431-450$.

4. D. Z. Du and F. K. Hwang, A proof of Gilbert-Pollak's conjecture on the Steiner ratio, Algorithmica 7 (1992), $121-135$. 
5. D. Z. Du and F. K. Hwang, Reducing the Steiner problem in a normed plane, SIAM J. Comput. 21 (1992), 1001-1007.

6. M. R. Garey, R. L. Graham, and D. S. Johnson, The complexity of computing Steiner minimal trees, SIAM J. Appl. Math. 32 (1977), 835-859.

7. M. R. Garey and D. S. Johnson, The rectilinear Steiner tree problem is NP-complete, SIAM J. Appl. Math. 32 (1977), 826-834.

8. E. N. Gilbert and H. O. Pollak, Steiner minimal trees, SIAM J. Appl. Math. 178 (1968), 1-29.

9. F. K. Hwang, On Steiner minimal trees with rectilinear distance, SIAM J. Appl. Math. 30 (1976), 104-114.

10. G. Lawlor and F. Morgan, Paired calibrations applied to soapfilms, immiscible fluids, and surfaces or networks minimizing other norms, Preprint (1991).

11. Z. C. Liu and D. Z. Du, On Steiner minimal trees with $L_{p}$ distance, Algorithmica 7 (1992), 179-191.

12. Z. A. Melzak, Companion to Concrete Mathematics, Vol. II, Wiley, New York, 1976.

13. D. Sankoff and $P$. Rousseau, Locating the vertices of a Steiner tree in an arbitrary metric space, Math. Programming 9 (1975), 240-246.

14. M. Sarrafzadeh and C. K. Wong, Hierarchical Steiner tree construction in uniform orientations, Unpublished manuscript.

15. J. M. Smith and M. Gross, Steiner minimal trees and urban service networks, J. Sociol. Econ. Planning 16 (1982), 21-38.

16. D. Cieslik, Das Steiner-Problem in Banach-Minkowski-Räumen, Habilitationsschrift, ErnstMoritz-Arnot-Universität, Greifswald, 1991.

Received October 4, 1991, and in revised form July 30, 1992. 